\title{
Efeito da Meditação Focada no Estresse e Mindfulness Disposicional em Universitários
}

\author{
Marina Xavier Carpena* \\ Universidade Federal do Rio Grande \\ Carolina Baptista Menezes \\ Universidade Federal de Santa Catarina
}

\begin{abstract}
RESUMO - Intervenções meditativas têm sido utilizadas nas universidades para reduzir estresse. Este trabalho objetivou investigar o efeito de um treino de meditação focada de seis semanas no estresse (utilizando o Inventário de Sintomas de Estresse da Lipp) e mindfulness disposicional (utilizando o Questionário das Cinco Facetas de Mindfulness versão Brasileira) em universitários. Um ensaio controlado não randomizado comparou um grupo experimental e um grupo de lista de espera antes e após a intervenção. Apenas o grupo experimental reduziu a presença de estresse nas fases resistência, quase-exaustão e geral. Apresentou, ainda, aumento significativo de mindfulness nas facetas descrever, não reagir e no escore total. Conclui-se que treinos meditativos podem configurar uma alternativa para intervenções visando ao manejo de estresse e aprimoramento de habilidades de mindfulness nas universidades.
\end{abstract}

Palavras-chave: meditação, estresse, mindfulness, universitários.

\section{Effects of Focused Meditation on Stress and Dispositional Mindfulness in College Students}

\begin{abstract}
Meditation based interventions have been used in universities to reduce stress. This study aimed to investigate the effect of a six-week focused meditation-training on stress (using Lipp's Inventory of Stress Symptoms) and dispositional mindfulness (using the Five Facet Mindfulness Questionnaire - Brazilian version) in college students from southern Brazil. A nonrandomized controlled trial was conducted to compare the experimental group and the waitlist control group before and after the training. Compared with controls, the experimental group demonstrated significant improvements for stress, and increases in mindfulness scores, particularly for the facets describe and non-react. The results suggest that meditative practice may be an alternative for interventions aiming to tackle stress and enhance mindfulness skills in the university context.
\end{abstract}

Keywords: meditation, stress, mindfulness, college students.

Tanto no Brasil (Cerchiari, 2004), quanto em outros países (Beiter et al., 2015), a fase universitária compreende um dos períodos do desenvolvimento que parece especialmente suscetível ao estresse e fatores relacionados (por exemplo, problemas de sono). Além disso, é no início da vida adulta, particularmente no período universitário, que muitos dos transtornos mentais têm maior chance de surgir pela primeira vez (Neves \& Dalgalarrondo, 2007). No estudo realizado por Neves e Dalgalarrondo (2007) com uma amostra de universitários brasileiros, entre os principais fatores associados à presença de transtornos mentais estavam pior relacionamento interpessoal, falta de apoio parental, sentimento de discriminação e problemas de uso de drogas na família. Portanto, condições estressoras de vida podem estar influenciando tanto o surgimento de problemas mentais, como a sua manutenção e agravamento.

Considerando os prejuízos de curto e longo prazo que o estresse pode exercer sobre o estudante, tanto do ponto de vista acadêmico, como pessoal, tem-se ressaltado a

* Endereço para correspondência: Faculdade de Medicina, Programa de Pós-Graduação em Saúde Pública da Universidade Federal do Rio Grande, FURG, Rua Gen. Osório s/n, Centro, Rio Grande, RS, Brasil. CEP 96.200-190.E-mail: marinacapena_@hotmail.com importância de as universidades continuamente avaliarem a saúde mental de seus alunos, bem como oferecerem intervenções voltadas às suas necessidades (Beiter et al., 2015). Programas baseados na meditação constituem uma modalidade de intervenção que vem sendo crescentemente aplicada no ambiente universitário com o objetivo de reduzir o estresse, bem como aumentar o bem-estar e a qualidade de vida (Fan, Tang, \& Posner, 2014; Greeson, Juberg, Maytan, James, \& Rogers, 2014; Jain et al., 2007; Menezes et al., 2013; Oman, Shapiro, Thoresen, Plante, \& Flinders, 2008; Regehr, Glancy, \& Pitts, 2013). No Brasil, um estudo realizado na Universidade Federal do Rio Grande do Sul demonstrou que existe um grande interesse por parte dos universitários em participarem deste tipo de intervenção (Menezes, Fiorentin, \& Araujo, 2012).

$\mathrm{Na}$ perspectiva da ciência psicológica, a prática meditativa, seja ela do tipo concentrativa ou atenção plena (mindfulness), tem sido definida como um treino sistemático da regulação atencional e emocional (para uma revisão dos tipos meditativos, ver Dahl, Lutz, \& Davidson, 2015). Segundo Dahl et al. (2015), por regulação atencional entende-se a capacidade de iniciar, direcionar e sustentar processos atencionais na consciência do momento presente, 
ao mesmo tempo em que se busca perceber e desengajar a atenção das distrações e dos processos auto-reflexivos. Já a regulação emocional sugere a capacidade de não reagir afetivamente aos conteúdos e experiências internas. Para estes autores, estas habilidades regulatórias caracterizam os potenciais mecanismos por trás da relação entre meditação e o desenvolvimento de uma maior estabilidade mental/ emocional (Dahl et al., 2015).

Algumas evidências do efeito positivo de intervenções baseadas na meditação sobre a redução do estresse têm sido obtidas em amostras universitárias (Regehr et al., 2013). Por exemplo, um estudo comparou o efeito de dois programas distintos de oito semanas de meditação (Programa de Redução de Estresse Baseado em Mindfulness [MBSR] e o Programa de Oito Pontos [EPP]) nos níveis de estresse com um grupo controle de lista espera em universitários saudáveis. Os resultados indicaram que apenas nos dois grupos que receberam as intervenções houve uma redução significativa no autorrelato de estresse (Oman et al., 2008). No estudo de Jain et al. (2007), uma intervenção de quatro semanas de meditação mindfulness, em comparação ao grupo de lista de espera, produziu uma redução significativa no autorrelato de estresse, relativo ao grupo controle. Mais recentemente, outra intervenção de quatro semanas de meditação mindfulness (Programa Koru) foi igualmente capaz de produzir uma redução significativa do autorrelato de estresse em uma amostra universitária não clínica, quando comparada a uma condição controle de lista de espera (Greeson et al., 2014).

Alguns dados têm sugerido que o aumento nas habilidades de mindfulness disposicional pode ser um dos mecanismos psicológicos pelos quais a meditação ajuda a reduzir o estresse. Por exemplo, no estudo de Greeson et al. (2014), apenas o grupo que recebeu a intervenção de meditação apresentou um aumento significativo dos escores de mindfulness geral. Ademais, neste grupo houve uma correlação negativa significativa entre o escore total de mindfulness e estresse percebido, sugerindo que o aumento de mindfulness disposicional relacionou-se com a diminuição da percepção de estresse.

Mindfulness disposicional compreende uma qualidade mental que as práticas meditativas buscam desenvolver. Apesar da falta de consenso sobre a natureza uni ou multidimensional do construto, de forma geral, enquanto uma habilidade mental, mindfulness refere-se a um estado cognitivo e afetivo de atenção plena, isto é, a uma consciência menos condicionada e automatizada, devido a uma maior capacidade de observar, descrever, aceitar, não julgar e não reagir (Baer et al., 2008).

No contexto de programas baseados em meditação, em consonância com o estudo de Greeson et al. (2014), o aumento no autorrelato de mindfulness disposicional também foi demonstrado por outras intervenções realizadas com amostras de universitários (Oman et al., 2008; Shapiro, Brown, Thoresen, \& Plante, 2011). Além disso, o estudo de Oman et al. (2008) encontrou que nos dois grupos que receberam dois tipos de intervenção de meditação (MBSR e EPP), o aumento em mindfulness disposicional mediou a redução do estresse percebido e da ruminação, corroborando a correlação observada por Gresson et al. (2014).
Do ponto de vista metodológico, destaca-se que uma característica dos programas anteriormente citados (MBSR, EPP, IBMT e Koru) é o uso da combinação de diferentes técnicas, tal como psicoeducação, exercícios de relaxamento, escaneamento corporal, imaginação guiada, entre outros. Portanto, é difícil concluir se o efeito dos treinos sobre os desfechos advém da técnica meditativa formal (em particular, a meditação sentada e silenciosa) ou da combinação destas diferentes técnicas. Neste sentido, uma contribuição relevante de dois estudos que utilizaram uma intervenção com seis encontros semanais foi demonstrar que efeitos psicológicos positivos, tal como melhor regulação emocional, foram produzidos pela técnica meditativa formal (meditação focada, sentada e silenciosa), já que nestes estudos não houve uma combinação de diferentes técnicas, exceto um breve período de respiração diafragmática que precedeu a prática de meditação em todos os encontros (Menezes \& Bizarro, 2015; Menezes et al., 2013). Contudo, os efeitos desta intervenção especificamente sobre as variáveis de estresse e mindfulness não foram avaliados.

Assim, o objetivo do presente trabalho baseou-se nas seguintes considerações: a relevância de testar intervenções que possam auxiliar na redução do estresse no contexto universitário; a relevância de testar o efeito de intervenções baseadas na meditação sobre mindfulness disposicioinal e a potencial relação entre o aumento de mindfulness disposicional e a redução do estresse; o fato de que estes desfechos não foram avaliados por uma intervenção de seis encontros baseada exclusivamente no treino da meditação focada formal.

Dessa forma, este trabalho buscou avaliar o efeito de seis semanas de um treino de meditação focada no desfecho primário de estresse e secundário de mindfulness disposicional em uma amostra universal de estudantes universitários, comparando a um grupo controle de lista de espera. Considerando que este trabalho seria realizado na Universidade Federal do Rio Grande, onde a intervenção aconteceria pela primeira vez, optou-se por inicialmente investigar uma amostra universal (isto é, com participantes sem e com sintomas psiquiátricos menores), para posteriormente poder avaliar a necessidade de adaptar a intervenção a demandas mais específicas. Esperava-se que após as seis semanas de treino, o grupo experimental apresentaria uma redução significativa da presença de estresse, bem como um aumento significativo dos escores de mindfulness disposicional, relativo ao grupo controle. Também era esperado que no pós-teste houvesse uma associação significativa entre um maior escore de mindfulness e um menor índice de estresse em ambos os grupos, mas com um tamanho de efeito maior no grupo experimental.

\section{Método}

\section{Participantes}

Este foi um ensaio controlado não randomizado com duração de seis semanas, com duas avaliações (pré e pósteste), realizado com uma amostra universal de estudantes 
universitários de graduação e pós-graduação da Universidade Federal do Rio Grande (FURG). Estes foram convidados a participar da pesquisa por meio de anúncios no site da FURG e por cartazes distribuídos pelo campus Carreiros da FURG. Um total de 138 estudantes demonstraram interesse em participar. Destes, foram excluídos aqueles que não tinham entre 20 e 40 anos; relataram algum problema neurológico; e relataram ter experiência prévia com meditação ou yoga. Os 64 participantes restantes foram distribuídos em dois grupos conforme a sua disponibilidade de horário: grupo de meditação $(\mathrm{ME}=30)$ e grupo controle de lista de espera $(\mathrm{LE}=$ $34)$, sendo que a amostra final compreendeu 56 participantes $(\mathrm{ME}=29 ; \mathrm{LE}=27)$ (Figura 1). Todas as perdas correspondem a pessoas que não compareceram na avaliação pós-teste. Nenhum indivíduo do grupo meditação foi excluído pelo número de faltas, uma vez que esta variável foi controlada nas análises a fim de propiciar uma análise intention to treat.

\section{Instrumentos}

Questionário Sociodemográfico. Desenvolvido para o presente estudo, este questionário foi utilizado com a finalidade de coletar dados pessoais, tais como idade, sexo, curso de graduação, semestre que está cursando, uso de medicação psicoativa, se faz psicoterapia, doenças neurológicas e experiência prévia com meditação ou yoga.

Critério De Classificação Econômica Brasil - CCEB (Associação Nacional De Empresas de Pesquisa, 2010). Instrumento baseado em um sistema de avaliação de pontos que tem como objetivo principal classificar indivíduos brasileiros de acordo com a sua classe econômica. Nesta escala, desenvolvida pela Associação Brasileira de Empresas de Pesquisa, a escolaridade do chefe da família vale de 0 a 5 pontos; os demais pontos são fornecidos pela quantidade de bens de consumo duráveis que a família possui (automóvel, televisão em cores, rádio, geladeira, freezer, máquina de lavar roupa etc.); pela quantidade de cômodos da casa, com ênfase nos banheiros; e pela presença e quantidade de empregadas domésticas mensalistas que trabalham na casa. A soma desses indicadores classifica as populações em classes, sendo a Classe A1 (de 42 a 46 pontos) de maior poder aquisitivo, seguida da Classe A2 (de 35 a 41 pontos), Classe B1 (de 29 a 34 pontos), Classe B2 (de 23 a 28 pontos), Classe C1 (de 18 a 22 pontos), Classe C2 (de 14 a 17 pontos), Classe D (de 8 a 13 pontos), e Classe $\mathrm{E}$ (de 0 a 7 pontos), representando o menor poder aquisitivo.

Self-Report Questionnaire - SRQ (Mari \& Williams, 1986). O SRQ consiste em 20 questões investigando a presença de sintomas psiquiátricos menores, bem como 3 questões investigando a presença de sintomas psicóticos através de respostas binárias de sim ou não. A versão validada no Brasil apresentou coeficientes de sensibilidade e especificidade de $83 \%$ e $80 \%$, respectivamente. Considerando que o presente estudo objetivou avaliar uma amostra de caráter universal (i.e., sem considerar a sintomatologia psiquiátrica como critério de exclusão), optou-se por medir os sintomas psiquiátricos, a fim de poder controlar esta variável nas análises dos desfechos, caso os grupos diferissem significativamente com relação à sintomatologia.
Inventário de Sintomas de Stress para Adultos de Lipp - ISSL (Lipp, 2005). Instrumento validado para a população brasileira $(\alpha=0.91)$, que tem como finalidade detectar a presença geral de estresse (independente da fase); identificar a fase do estresse na qual a pessoa se encontra (alerta, resistência, quase-exaustão ou exaustão). As fases do estresse estão divididas de forma temporal, em que alerta diz respeito aos sintomas apresentados nas últimas 24 horas; resistência e quase-exaustão referem-se aos sintomas experimentados no período da última semana; e exaustão descreve os sintomas apresentados no último mês. No ISSL, o diagnóstico positivo de estresse é dado a partir da soma dos sintomas de cada fase, sendo que ao ultrapassar o número limite de qualquer uma das fases (alerta $>6$; resistência ou quase-exaustão $>3$ ou $>9$; exaustão $>8$ ), a pontuação indicará que a pessoa tem estresse. Portanto, as variáveis deste instrumento foram analisadas como categóricas, considerando a presença ou ausência de estresse em cada fase ou de forma geral.

Questionário das Cinco Facetas de Mindfulness FFMQ-BR (Barros, Kozasa, Souza, \& Ronzani, 2014). Adaptado para o Brasil, o FFMQ-BR é um questionário de 39 itens, que avalia as habilidades de mindfulness disposicional como um construto multifacetado. As respostas são fornecidas em uma escala likert de 5 pontos que variam de 1 (nunca ou raramente verdadeiro) à 5 (quase sempre ou sempre verdadeiro). A sua interpretação pode ser feita através de 5 facetas: Observar (perceber e estar atento aos eventos e experiências; $\alpha=0.76$ ); Descrever (capacidade de nomear e descrever as experiências observadas; $\alpha=0.76$ ); Agir com consciência (prestar atenção às atividades em andamento, ao invés de realizá-las mecanicamente com a atenção em outro lugar; $\alpha=0.79$ ); Não julgar (não avaliar ou utilizar juízo de valor para os pensamentos e emoções; $\alpha=0.78$ ); e Não Reagir (capacidade de experimentar sentimentos e pensamentos sem reagir ou ser influenciado pelos mesmos; $\alpha=0.68$ ); ou com base no escore total $(\alpha=0.81)$, o qual indica uma habilidade geral de atenção plena. Neste instrumento, os desfechos foram analisados como variáveis contínuas.

\section{Procedimentos}

Coleta de Dados. Após a divulgação da pesquisa, aqueles interessados em participar responderam ao Termo de Consentimento Livre e Esclarecido, ao questionário sociodemográfico e ao SRQ em formato online. Os participantes elegíveis foram contatados para o agendamento da primeira testagem, após a qual foram distribuídos de forma não randômica (isto é, por conveniência, conforme sua disponibilidade de horários) em um dos dois grupos (meditação ou lista de espera). As duas avaliações (préteste e pós-teste) aconteceram durante as duas semanas que antecederam e sucederam as seis semanas de intervenção, respectivamente, tendo uma duração aproximada de 45 minutos. Os participantes do grupo controle não tiveram nenhuma atividade durante o período da intervenção, sendo que aqueles interessados receberam a intervenção após o término da coleta de pós-teste.

Treino de meditação. $O$ treino de meditação baseouse no estudo de Menezes et al. (2013). O mesmo incluiu 
seis encontros semanais consecutivos com uma duração aproximada de uma hora e quinze minutos. O treino foi conduzido por um dos membros da equipe de pesquisa, a qual além de ter experiência pessoal com a prática de meditação concentrativa, passou por treinamento para replicar a intervenção. Os encontros contaram com a seguinte estrutura de atividades: conversa em grupo envolvendo dúvidas, dificuldades, bem como relatos de experiências; informações e instruções para as práticas; a prática de exercícios preparatórios, que compreendiam breves alongamentos para preparar o corpo para a meditação formal ( $\sim 5$ minutos); execução de exercícios de respiração diafragmática $(\sim 5$ minutos); realização da prática formal (meditação focada sentada e silenciosa); realização de variações de práticas meditativas (caminhada meditativa e comer meditativo); fechamento com mais um tempo para conversas e trocas de experiências. Nos dois primeiros encontros, a prática formal durou 15 e 20 minutos, respectivamente. Nos quatro encontros restantes a prática foi de 30 minutos. A prática de caminhada meditativa ocorreu no $3^{\circ}$ e $4^{\circ}$ encontro $(\sim$ 5 minutos e 10 minutos, respectivamente), enquanto comer meditativo foi no $5^{\circ}$ e $6^{\circ}$ (ambos de $\sim 10$ minutos). Os participantes poderiam sentar em um colchonete de pernas cruzadas, ou em uma cadeira sem encostar as costas e com os pés bem apoiados no chão. Independente de estarem no chão ou na cadeira, os participantes poderiam trocar a opção de acento quando quisessem. No caso de desconforto, os participantes podiam se movimentar para melhor acomodar o corpo, mas eram instruídos a ser discretos e silenciosos a fim de não perturbar os demais. Nos exercícios de respiração, todos foram instruídos a controlar os movimentos da respiração, expandindo e retraindo o abdômen na inspiração e expiração, respectivamente. Também foram solicitados a prestar atenção e a ampliar a percepção dos movimentos abdominais de cada fase da respiração, tentando prolongar a exalação levemente. Para a meditação, enfatizou-se que nenhum esforço particular deveria ser feito para respirar. No entanto, a fim de caracterizar a meditação da atenção focada, bem como possibilitar que os participantes novatos mantivessem o foco da atenção na respiração, os mesmos foram instruídos a contar mentalmente cada expiração (ressalta-se que outros tipos de foco, tal como mantras, não foram utilizados para evitar qualquer ligação com tradições filosóficas ou espirituais). Nos três primeiros encontros a contagem compreendeu ciclos de 1 à 10 , e nos três últimos encontros os participantes faziam uma contagem regressiva de 100 à 1 (sempre um número por exalação). Em ambas as contagens, os participantes eram instruídos a recomeçar o ciclo do princípio (número 1, ou número 100) caso percebessem que haviam se distraído e perdido a contagem.

Análise de dados. Primeiramente foram realizadas análises descritivas dos dados obtidos nos questionários Sociodemográfico, ABEP, SRQ-20, ISSL e FFMQ. Posteriormente foram realizados os testes $t$ de Student para amostras independentes e Exato de Fisher para comparar os grupos controle e intervenção na linha de base (pré-teste). Para avaliar os efeitos da intervenção (termo de interação) para os desfechos primários e secundários, considerando os fatores grupo (Grupo Meditação x Grupo Controle) e tempo (pré-teste x pós-teste), foi realizada a Generalized Estimating
Equations (GEE) com estimador de variância robusto, controlando para $n^{\circ}$ de faltas do grupo da intervenção. Esta análise foi realizada de acordo com o princípio da intenção de tratar, utilizando família binomial e função de ligação logito (equivalente à regressão logística) para os desfechos dicotômicos (estresse em cada fase e presença geral), e família gaussiana e função de ligação identidade (equivalente à regressão linear) para as variáveis numéricas (escores de FFMQ em cada domínio e total) (Edwards, 2000). Para interpretar a análise GEE, é importante considerar que são utilizados parâmetros na equação para obter o resultado do desfecho. Os principais parâmetros utilizados são $\beta 1, \beta 2$ e $\beta 3$, que se referem aos coeficientes beta da GEE. O primeiro coeficiente, $\beta 1$, é referente à comparação dos grupos no préteste; o segundo, $\beta 2$, é referente ao efeito do tempo no grupo controle, ou seja, compara-se os dados do pré e pós-teste do grupo controle; e $\beta 3$ refere-se à interação entre Tempo e Grupo, em que é comparada a mudança do pré e pós-teste, considerando ambos os grupos. Também foi realizada uma análise de sensibilidade para avaliar o impacto dos dados faltantes nas análises para os desfechos primários (presença de estresse nas diferentes fases e no geral). Para tanto, foram simuladas todas as situações que poderiam ocorrer (presença ou ausência dos desfechos) tendo em vista o número de dados faltantes em cada grupo. Além disso, foi realizada a Correlação de Pearson parcial dos desfechos de estresse e mindfulness disposicional que apresentaram mudança significativa na GEE com o número de faltas do grupo meditação, controlando os escores pré-teste. Correlações parciais também foram realizadas entre estes desfechos para cada grupo, controlando os escores do pré-teste. Todas as análises foram realizadas no software estatístico STATA 14, adotando um nível de significância bicaudal de 0.05.

\section{Considerações Éticas}

O presente estudo foi aprovado pelo Comitê de Ética em Pesquisa na Área da Saúde da Universidade Federal do Rio Grande (CEPAS/FURG), sob o registro n 1182014.

\section{Resultados}

\section{Pré-Teste}

$\mathrm{Na}$ linha de base (pré-teste) os grupos não diferiram quanto às médias de idade $\left[M_{\text {meditação }}=25.1(D P=5.8)\right.$; $\left.M_{\text {controle }}=26.6(D P=9.9)\right]$ e escores do SRQ-20 $\left[M_{\text {meditação }}=\right.$ $\left.8.7(D P=4) ; M_{\text {controle }}=9(D P=5.9)\right]$. Não houve diferenças significativas nas distribuições de sexo $(p=0.772)$ e classe socioeconômica $(p=0.571)$ entre grupos. A frequência do uso de medicação $\left(F_{\text {meditacão }}=10 \% ; F_{\text {controle }}=11.8 \%\right)$ e de psicoterapia $\left(F_{\text {meditação }}=20 \% ; F_{\text {controle }}=20.6 \%\right)$ foi pequena e não diferiu entre os grupos (Tabela 1). Deste modo, nenhuma destas variáveis foi controlada nas análises posteriores. Os desfechos da presença de estresse (em cada fase e geral) e mindfulness disposicional (em cada faceta e total) também não diferiram entre os grupos no pré-teste tanto nas análises 


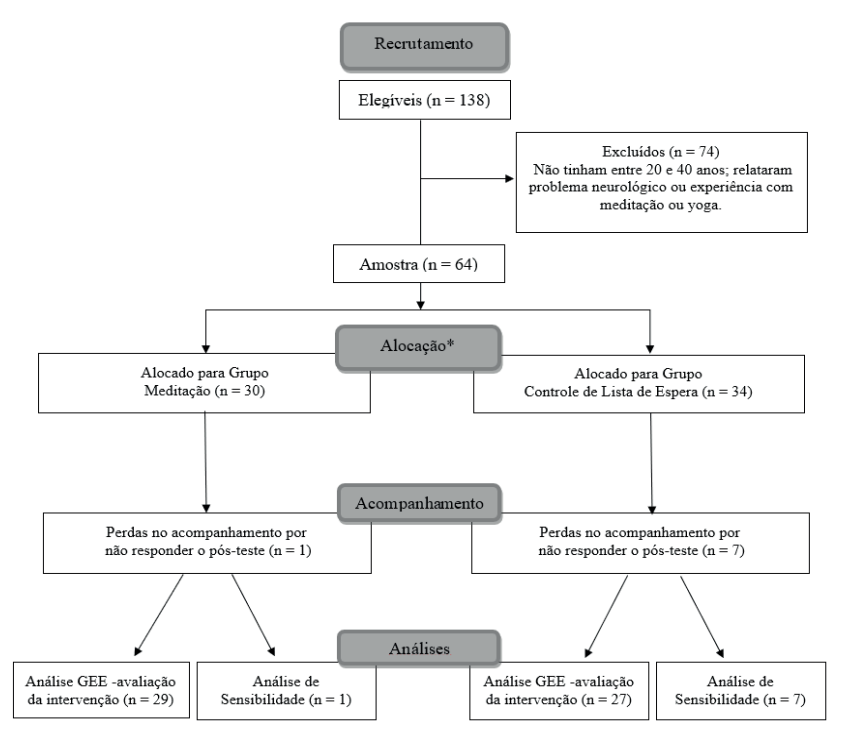

Figura 1. Amostragem e fluxo de participantes do ensaio controlado não randomizado.

Nota. *A alocação foi realizada de acordo com a disponibilidade de horário dos participantes.

de Teste te Teste Exato de Fisher (valores de $p>0.05$; Tabela 1), quanto nas análises GEE (valor de $\beta 1$; Tabela 3 e 4).

\section{Pós-teste}

Ao término da intervenção houve apenas uma perda no grupo de intervenção (indivíduo que não respondeu o pósteste), totalizando um $n=29$ no pós-teste. A média de faltas do grupo meditação foi de $1.55(D P=1.57 ;$ Min. $=0 ;$ Máx. $=5)$, sendo que 10 (34.5\%) participantes compareceram em todos os encontros, 5 (17.2\%) compareceram a cinco encontros, 9 (31.1\%) estiveram em quatro encontros, $3(10.3 \%)$ foram a três encontros, e $2(6.9 \%)$ participaram de um encontro. Na Tabela 2 podem ser observadas as frequências absoluta e relativa da presença de estresse em cada fase e no geral. Nesta tabela também são apresentadas as médias, acompanhadas de seus respectivos desvios padrão dos escores de FFMQ em cada faceta e do escore total.

GEE do desfecho Estresse. Para os desfechos de estresse (Tabela 3), quando foram comparados os resultados dos pré e pós-teste apenas do grupo controle ( $\beta 2)$, não foram observadas diferenças significativas (EstresseResistência $p$ $=0.100$; EstresseQuaseexausão $p=0.298$; EstresseExaustão $p=0.102$; EstresseTotal $p=0.939$ ). Estes dados indicam que apenas o fato de as pessoas do grupo controle terem participado do estudo não resultou em mudanças significativas do desfecho e que, portanto, não houve efeito principal do tempo. Cabe destacar que nesta análise não está presente a fase de alerta, pois no pós-teste de ambos os grupos não houve nenhum indivíduo com pontuação nesta fase. Quando o modelo de análise considerou os fatores grupo vs. tempo ( $\beta 3)$, observou-se que a intervenção de meditação produziu uma redução significativa dos sintomas de estresse nas fases resistência $(p=0.023)$ e quase-exaustão $(p<0.001)$,
Tabela 1. Apresentação e comparação de médias e porcentagens dos participantes dos Grupos Meditação e Controle na linha de base

\begin{tabular}{|c|c|c|c|}
\hline Variáveis & $\mathrm{ME}^{1}$ & $\mathrm{LE}^{2}$ & $p$ \\
\hline Idade & $25.1(5.8)$ & $26.6(9.9)$ & $0.488 \dagger$ \\
\hline SRQ & $8.7(4)$ & $9(5.9)$ & $0.858+$ \\
\hline Sexo Feminino & $23(76.7 \%)$ & $25(73.5 \%)$ & $0.772 \#$ \\
\hline Medicação Psicoativa & $3(10 \%)$ & $4(11.8 \%)$ & $0.821 \#$ \\
\hline Psicoterapia & $6(20 \%)$ & $7(20.6 \%)$ & $0.953 \#$ \\
\hline $\mathrm{ABEPa}$ & & & $0.571 \#$ \\
\hline A & $3(10 \%)$ & $3(9,38 \%)$ & \\
\hline $\mathrm{B}$ & $9(30 \%)$ & $12(37,5 \%)$ & \\
\hline $\mathrm{C}$ & $14(46,7 \%)$ & $16(50 \%)$ & \\
\hline $\mathrm{D}$ & $4(13,3 \%)$ & $1(3,13 \%)$ & \\
\hline \multicolumn{4}{|l|}{ Estresse } \\
\hline Alerta & $5(16,7 \%)$ & $3(8,8 \%)$ & $0.458 \#$ \\
\hline Resistência & $15(50 \%)$ & $21(61,7 \%)$ & $0.450 \#$ \\
\hline Quase-resistência & $3(10 \%)$ & $4(11,7 \%)$ & $1.000 \#$ \\
\hline Exaustão & $5(16,7 \%)$ & $7(20,6 \%)$ & $0.757 \#$ \\
\hline Geral & $16(53,3 \%)$ & $21(61,8 \%)$ & $0.613 \#$ \\
\hline \multicolumn{4}{|l|}{ Mindfulness Disp. } \\
\hline Observar & $3.44(.97)$ & $3.16(.85)$ & $0.224 \dagger$ \\
\hline Descrever & $3.20(.82)$ & $3.47(1.05)$ & $0.268 \dagger$ \\
\hline Agir com consc. & $3.10(.85)$ & $3.16(.84)$ & $0.787 \dagger$ \\
\hline Não julgar & $3.22(.99)$ & $3.16(.89)$ & $0.779 \dagger$ \\
\hline Não reagir & $2.65(.63)$ & $2.75(.76)$ & $0.538 \dagger$ \\
\hline Total & $3.12(.48)$ & $3.14(.56)$ & $0.891 \dagger$ \\
\hline
\end{tabular}

Nota $. \mathrm{ME}=$ meditação; $\mathrm{LE}=$ lista de espera; As médias das variáveis numéricas estão acompanhadas de seus desvios padrão entre parênteses; As variáveis categóricas são apresentadas em frequência absoluta e frequência relativa entre parênteses;

${ }^{1}$ Número amostral $=30 ;{ }^{2}$ Número amostral $=34$; †valor de $\mathrm{p}$ do Teste t; \# valor de p do teste Exato de Fisher; ${ }^{a}$ dois participantes do grupo controle não responderam este questionário, tornando o "n" para esta variável $=32$.

bem como da presença geral ( $p=0.002)$. Conforme indica a Razão de Odds (RO), o treino meditativo conferiu uma proteção de, respectivamente, 78.4\% (IC95\% 0.058; 0.812), 99.9\% (IC95\% 6.2x10-7; 2.8x10-4) e 89.9\% (IC95\% 0.024; 0.427 ) para a presença de estresse nas fases de resistência, quase-exaustão e geral.

GEE do desfecho Mindfulness Disposicional. Quando foram comparados os resultados do pré e pós-testes dos desfechos de mindfulness disposicional (Tabela 4) apenas do grupo controle $(\beta 2)$, não foram observadas diferenças significativas $\left(\mathrm{FFMQ}_{\mathrm{Observar}} p=0.869 ; \mathrm{FFMQ}_{\text {Descrever }} p=\right.$ $0.090 ; \mathrm{FFMQ}_{\mathrm{Agircomconsc}} \cdot p=0.901 ; \mathrm{FFMQ}_{\text {Nãoulgar }} p=0.228$; $\left.\mathrm{FFMQ}_{\text {Nãoreagir }} p=0.873 ; \mathrm{FFMQ}_{\text {ToTaL }} p=0.316\right)$. Ou seja, a GEE indicou que apenas a participação do grupo controle no estudo não produziu mudanças significativas no desfecho e que, portanto, não houve efeito principal de tempo. No modelo de análise que considerou os fatores grupo vs. tempo ( $\beta 3)$, observou-se que o termo de interação passou a ser significativo, indicando que no grupo experimental houve 
Tabela 2. Apresentação descritiva dos desfechos de Estresse e de Mindfulness disposicional nos Grupos Meditação e Controle no pré e pós-teste

\begin{tabular}{|c|c|c|c|c|}
\hline & \multicolumn{2}{|c|}{ Grupo Meditação } & \multicolumn{2}{|c|}{ Grupo Controle } \\
\hline & $\begin{array}{c}\text { Pré-Teste } \\
(\mathrm{N}=30)\end{array}$ & $\begin{array}{c}\text { Pós-Teste( } \\
\mathrm{N}=29)\end{array}$ & $\begin{array}{l}\text { Pré-Teste } \\
(\mathrm{N}=34)\end{array}$ & $\begin{array}{l}\text { Pós-Teste(N } \\
=27)\end{array}$ \\
\hline Questionários & $\begin{array}{l}\text { M(DP) } \\
\text { F (\%) }\end{array}$ & $\begin{array}{c}\text { M(DP) } \\
F(\%)\end{array}$ & $\begin{array}{l}\text { M(DP) } \\
\text { F (\%) }\end{array}$ & $\begin{array}{l}\mathrm{M}(\mathrm{DP}) \\
\mathrm{F}(\%)\end{array}$ \\
\hline ISSL & & & & \\
\hline Alerta & $5(16.7 \%)$ & $0(0 \%)$ & $3(8.8 \%)$ & $0(0 \%)$ \\
\hline Resistência & $15(50 \%)$ & $3(10.3 \%)$ & $21(61.8 \%)$ & $13(48.1 \%)$ \\
\hline $\begin{array}{l}\text { Quase- } \\
\text { exaustão }\end{array}$ & $3(10 \%)$ & $0(0 \%)$ & $4(11.8 \%)$ & $1(3.7 \%)$ \\
\hline Exaustão & $5(16,7 \%)$ & $2(6,9 \%)$ & $7(20.6 \%)$ & $3(11.1 \%)$ \\
\hline Geral & $16(53.3 \%)$ & $3(10.3 \%)$ & $21(61.8 \%)$ & $17(63 \%)$ \\
\hline FFMQ & & & & \\
\hline Observar & $3.37(0.95)$ & $3.84(0.65)$ & $3.14(0.89)$ & $3.17(0.93)$ \\
\hline Descrever & $3.32(0.75)$ & $3.65(0.84)$ & $3.64(0.97)$ & $3.37(0.99)$ \\
\hline $\begin{array}{l}\text { Agir com } \\
\text { Consciência }\end{array}$ & $3.12(0.90)$ & $3.39(0.73)$ & $3.34(0.73)$ & $3.27(0.27)$ \\
\hline Não Julgar & $3.21(0.96)$ & $3.49(0.71)$ & $3.17(0.78)$ & $2.99(0.79)$ \\
\hline Não Reagir & $2.71(0.13)$ & $2.75(0.13)$ & $3.03(0.12)$ & $2.77(0.12)$ \\
\hline Total & $3.15(0.49)$ & $3.21(0.51)$ & $3.47(0.46)$ & $3.11(0.49)$ \\
\hline
\end{tabular}

Nota. $M=$ Médias; $D P=$ Desvio padrão; $\mathrm{F}=$ frequência absoluta; $\%=$ frequência relativa. introduzidos por dados faltantes. Ou seja, para diferentes cenários de dados faltantes é rodada uma análise simulando estes dados como tendo ou não o desfecho (Tabela 5), a fim de avaliar se nestes cenários se manteria o efeito encontrado na análise GEE. Nos resultados da fase de resistência pode-se observar que a significância estatística se manteve em dez das 14 situações possíveis. Além disso, todas as estimativas pontuais de efeito (RO) se mantiveram no mesmo sentido quando comparados com a análise original. Já na fase de quase-exaustão, a significância estatística não foi mantida em nenhuma situação. No entanto, apenas uma das estimativas pontuais de efeito ( $\mathrm{RO}$ ) apresentou um sentido oposto ao observado na análise original, ou seja, apenas na situação simulada em que nenhum dos sete participantes do grupo controle apresentaria estresse na fase de quase-exaustão a RO expressa um efeito não protetor. Quando a análise de sensibilidade é feita para a presença geral de estresse, a significância estatística é mantida em todos os cenários simulados. Além disso, o efeito protetivo da intervenção encontrado nessa análise corrobora o efeito encontrado nas análises GEE. Em suma, as análises de sensibilidade indicaram que os dados faltantes não impactariam os resultados obtidos das análises GEE e mesmo nos cenários mais desfavoráveis o efeito protetivo da intervenção se manteve, exceto em uma situação da fase de quase-exaustão.

Tabela 3. Análises GEE (família binomial e função de ligação logito) envolvendo desfechos binários obtidos pelo ISSL

\begin{tabular}{|c|c|c|c|c|c|}
\hline Desfecho & Termo & $\mathrm{RO}^{\mathrm{a}}$ & $\mathrm{EP}^{\mathrm{b}}$ & IC95\% & $p$ \\
\hline \multirow[t]{3}{*}{ Resistência } & Grupos $\uparrow$ & 0.619 & 0.317 & $0.227 ; 1.688$ & 0.349 \\
\hline & Controlet† & 0.562 & 0.197 & $0.283 ; 1.117$ & 0.100 \\
\hline & Grupo x Tempo\# & 0.216 & 0.146 & $0.058 ; 0.812$ & 0.023 \\
\hline \multirow[t]{3}{*}{ Quase exaustão } & Grupos $\dagger$ & 0.833 & 0.679 & $0.168 ; 4.116$ & 0.823 \\
\hline & Controlet† & 0.297 & 0.346 & $0.030 ; 2.920$ & 0.298 \\
\hline & Grupo x Tempo\# & $1.3 \times 10-5$ & $2.1 \times 10-5$ & $6.2 \times 10-7 ; 2.8 \times 10-4$ & $<0.001$ \\
\hline \multirow[t]{3}{*}{ Exaustão } & Grupos $\dagger$ & 0.771 & 0.504 & $0.214 ; 2.774$ & 0.691 \\
\hline & Controlet† & 0.443 & 0.220 & $0.167 ; 1.175$ & 0.102 \\
\hline & Grupo x Tempo\# & 1.020 & 0.690 & $0.271 ; 3.842$ & 0.977 \\
\hline \multirow[t]{3}{*}{ Geral } & Grupos $\dagger$ & 0.707 & 0.363 & $0.259 ; 1.931$ & 0.499 \\
\hline & Controlet† & 1.034 & 0.450 & $0.441 ; 2.426$ & 0.939 \\
\hline & Grupo x Tempo\# & 0.101 & 0.074 & $0.024 ; 0.427$ & 0.002 \\
\hline
\end{tabular}

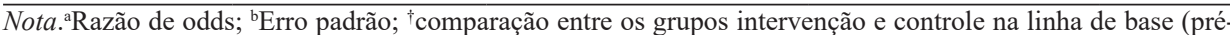
testes) ( $\beta 1)$; ${ }^{\dagger \dagger}$ comparação dos resultados do pós-teste e pré-teste, considerando apenas o grupo controle ( $\left.\beta 2\right)$ "comparação dos grupos intervenção e controle quanto a mudanças entre os resultados do pós-teste e do pré-teste $(\beta 3)$.

um aumento nos escores de FFMQ nas facetas descrever $(p=0.001)$ e não reagir $(p=0.040)$, assim como no escore total $(p=0.001)$. De acordo com o coeficiente do termo de interação, o aumento observado no grupo intervenção foi, respectivamente, em média de 0.586 (IC95\% 0.226; 0.946), 0.322 (IC95\% 0.015; 0.629) e 0.371 (IC95\% 0.147; 0.596) para faceta descrever e faceta não reagir e FFMQ total (em comparação ao grupo controle).

Análise de sensibilidade do Desfecho Estresse. Esta análise testa os resultados contra possíveis vieses
Correlações entre os desfechos e o número de faltas no Grupo Meditação. Foram realizadas correlações parciais entre $\mathrm{n}^{\circ}$ de faltas do grupo meditação e os desfechos estresse e mindfulness, controlando para os escores do pré-teste. Nenhuma destas correlações obteve significância estatística $(p>0.05)$. Nesse sentido, não foi necessário controlar para o $\mathrm{n}^{\mathrm{o}}$ de faltas nas correlações posteriores.

Correlações entre Estresse e Mindfulness para Ambos os Grupos. Foram realizadas correlações parciais entre 
Tabela 4. Análises GEE (família gaussiana e função de ligação identidade) com os dados obtidos pelo $F F M Q$

\begin{tabular}{|c|c|c|c|c|c|}
\hline FFMQ & Termo & Coef. & $\mathrm{EPb}$ & IC $95 \%$ & $p$ \\
\hline \multirow[t]{3}{*}{ Observar } & Grupos $\dagger$ & 0.279 & 0.227 & $-0.166 ; 0.724$ & 0.220 \\
\hline & Controle $\uparrow \dagger$ & 0.022 & 0.135 & $-0.243 ; 0.287$ & 0.869 \\
\hline & Grupo x Tempo\# & 0.389 & 0.214 & $-0.030 ; 0.808$ & 0.069 \\
\hline \multirow[t]{3}{*}{ Descrever } & Grupos $†$ & -0.266 & 0.233 & $-0.722 ; 0.190$ & 0.252 \\
\hline & Controle $\uparrow \dagger$ & -0.218 & 0.129 & $-0.470 ; 0.034$ & 0.090 \\
\hline & Grupo x Tempo\# & 0.586 & 0.184 & $0.226 ; 0.946$ & 0.001 \\
\hline \multirow[t]{3}{*}{ Agir com Consciência } & Grupos† & -0.576 & 0.211 & $-0.471 ; 0.356$ & 0.785 \\
\hline & Controle $\uparrow \dagger$ & 0.012 & 0.098 & $-0.179 ; 0.203$ & 0.901 \\
\hline & Grupo x Tempo\# & 0.269 & 0.173 & $-0.069 ; 0.607$ & 0.119 \\
\hline \multirow[t]{3}{*}{ Não julgar } & Grupos $\dagger$ & 0.063 & 0.226 & $-0.379 ; 0.506$ & 0.779 \\
\hline & Controle $\uparrow \dagger$ & -0.175 & 0.145 & $-0.461 ; 0.110$ & 0.228 \\
\hline & Grupo x Tempo\# & 0.313 & 0.219 & $-0.117 ; 0.743$ & 0.154 \\
\hline \multirow[t]{3}{*}{ Não reagir } & Grupos $\dagger$ & -0.109 & 0.172 & $-0.446 ; 0.228$ & 0.527 \\
\hline & Controlet† & 0.019 & 0.119 & $-0.214 ; 0.253$ & 0.873 \\
\hline & Grupo x Tempo\# & 0.322 & 0.157 & $0.015 ; 0.629$ & 0.040 \\
\hline \multirow[t]{3}{*}{ Total } & Grupos† & -0.018 & 0.129 & $-0.271 ; 0.245$ & 0.889 \\
\hline & Controle $\uparrow \dagger$ & -0.0639 & 0.064 & $-0.189 ; 0.061$ & 0.316 \\
\hline & Grupo x Tempo\# & 0.371 & 0.115 & $0.147 ; 0.596$ & 0.001 \\
\hline
\end{tabular}

Nota.$^{b}$ Erro padrão; ${ }^{\dagger}$ comparação entre os grupos intervenção e controle na linha de base (pré-testes)( $\left.\beta 1\right) ;{ }^{\dagger \dagger}$ comparação dos resultados do pós-teste e pré-teste, considerando apenas o grupo controle ( $\beta 2)$; ${ }^{*}$ comparação dos grupos intervenção e controle quanto a mudanças entre os resultados do pós-teste e do pré-teste $(\beta 3)$.

os desfechos que apresentaram mudança significativa na análise GEE (estresse: fases de resistência, quase-exaustão e presença geral de estresse; mindfulness: facetas descrever, não reagir e escore total), controlando para os escores do pré-teste. Foram encontradas correlações significativas entre a presença de estresse na fase de resistência e FFMQ total para ambos os grupos $\left(\mathrm{r}_{\text {meditacão }}=-0.657 ; \mathrm{p}_{\text {meditação }}<0.001\right.$; $\left.r_{\text {controle }}=-0.526 ; p_{\text {controle }}=0.007\right)$; entre presença geral de estresse e faceta descrever apenas para o grupo meditação (rmeditação $=-0.717 ; p_{\text {meditação }}<0.001 ; r_{\text {controle }}=-0.331 ; p_{\text {controle }}$ $=0.106)$ e entre presença geral de estresse e FFMQ total para ambos os grupos $\left(\mathrm{r}_{\text {meditacão }}=-0.693 ; \mathrm{p}_{\text {meditação }}<0.001\right.$; $\left.\mathrm{r}_{\text {controle }}=-0.537 ; \mathrm{pc}_{\text {ontrole }}=0.005\right)$. A correlação parcial entre as facetas descrever e não reagir, controlando para os escores pré-teste, foi significativa para o grupo meditação $(r=0.380$; $p=0.050)$, mas não para o grupo controle $(r=0.065 ; p=$ 0.757). As correlações com a fase quase exaustão não foram significativas $(p>0.05)$ para nenhum dos grupos.

\section{Discussão}

O objetivo principal deste estudo foi avaliar o efeito de uma intervenção de seis encontros semanais de meditação focada na presença de estresse em uma amostra universal de estudantes de graduação e pós-graduação, em comparação a um grupo controle de lista de espera. Também objetivouse avaliar o impacto da intervenção nas habilidades de mindfulness, bem como na relação entre os desfechos. Os resultados indicaram que em comparação ao grupo controle, na intervenção 1) houve uma redução estatisticamente significativa do estresse nas fases de resistência e quaseexaustão, bem como na presença geral de estresse; 2) houve um aumento estatisticamente significativo dos escores de mindfulness nas facetas descrever e não reagir, assim como no escore total; 3) as análises de sensibilidade indicaram que as perdas não teriam impactado a significância dos resultados obtidos nas análises GEE e que o efeito protetivo da intervenção para o estresse (desfecho primário) se manteria em outros possíveis cenários analisados (exceto em uma situação da fase de quase-exaustão); e 4) houve uma correlação negativa e significativa entre presença geral de estresse vs. faceta descrever (FFMQ), e uma correlação positiva e significativa entre as facetas descrever vs. não reagir (FFMQ). Para ambos os grupos, porém com um efeito maior para o grupo da meditação, houve correlação negativa e significativa entre fase de resistência do estresse vs. escore total de mindfulness, e entre presença geral de estresse vs. escore total de mindfulness.

$\mathrm{O}$ achado de que uma intervenção baseada na meditação pode auxiliar na redução dos níveis percebidos de estresse está em consonância com outros estudos que avaliaram tanto amostras universitárias (Carmody \& Baer, 2008; Fan et al., 2014; Greeson et al., 2014; Jain et al., 2007; Oman et al., 2008; Shapiro et al., 2011), como não universitárias (Baer, Carmody, \& Hunsinger, 2012). No presente estudo, a intervenção de meditação focada produziu um efeito protetivo para o estresse especialmente nas fases de resistência e quaseexaustão. Este é um dado interessante, visto que assim como em outras amostras universitárias brasileiras (Aguiar, Vieira, 
Tabela 5. Análises de sensibilidade para desfechos de estresse (termo Intervenção x Tempo

\begin{tabular}{|c|c|c|c|c|c|c|}
\hline \multirow[t]{2}{*}{$\begin{array}{l}\text { Desfecho } \\
\text { Estresse }\end{array}$} & \multicolumn{2}{|c|}{$\begin{array}{l}\mathrm{N} \text { de dados faltantes imputados como } \\
\text { tendo o desfecho }\end{array}$} & \multirow[t]{2}{*}{$\mathrm{RO}^{\mathrm{b}}$} & \multirow[t]{2}{*}{ Erro padrão } & \multirow[t]{2}{*}{ IC95\% } & \multirow[t]{2}{*}{ Valor $\mathrm{p}$} \\
\hline & $\mathrm{ME}$ & LE & & & & \\
\hline \multirow[t]{16}{*}{ Resistência } & 0 & 0 & 0.290 & 0.203 & $0.074 ; 1.142$ & 0.077 \\
\hline & & 1 & 0.256 & 0.177 & $0.066 ; 0.993$ & 0.049 \\
\hline & & 2 & 0.227 & 0.155 & $0.060 ; 0.866$ & 0.030 \\
\hline & & 3 & 0.202 & 0.141 & $0.052 ; 0.790$ & 0.022 \\
\hline & & 4 & 0.179 & 0.127 & $0.045 ; 0.721$ & 0.015 \\
\hline & & 5 & 0.160 & 0.112 & $0.040 ; 0.631$ & 0.009 \\
\hline & & 6 & 0.142 & 0.098 & $0.036 ; 0.551$ & 0.005 \\
\hline & & 7 & 0.126 & 0.089 & $0.031 ; 0.502$ & 0.003 \\
\hline & 1 & 0 & 0.401 & 0.254 & $0.116 ; 1.387$ & 0.149 \\
\hline & & 1 & 0.355 & 0.221 & $0.105 ; 1.204$ & 0.097 \\
\hline & & 2 & 0.315 & 0.193 & $0.094 ; 1.049$ & 0.060 \\
\hline & & 3 & 0.280 & 0.176 & $0.081 ; 0.959$ & 0.043 \\
\hline & & 4 & 0.249 & 0.160 & $0.070 ; 0.877$ & 0.030 \\
\hline & & 5 & 0.221 & 0.140 & $0.064 ; 0.766$ & 0.017 \\
\hline & & 6 & 0.196 & 0.123 & $0.058 ; 0.668$ & 0.009 \\
\hline & & 7 & 0.174 & 0.111 & $0.050 ; 0.610$ & 0.006 \\
\hline \multirow[t]{8}{*}{ Quase-exaustão $^{c}$} & 1 & 0 & 1.366 & 2.329 & $0.048 ; 38.650$ & 0.855 \\
\hline & & 1 & 0.662 & 1.027 & $0.032 ; 13.82$ & 0.790 \\
\hline & & 2 & 0.428 & 0.640 & $0.023 ; 8.027$ & 0.570 \\
\hline & & 3 & 0.310 & 0.456 & $0.017 ; 5.522$ & 0.426 \\
\hline & & 4 & 0.240 & 0.349 & $0.014 ; 4.138$ & 0.326 \\
\hline & & 5 & 0.193 & 0.279 & $0.011 ; 3.263$ & 0.254 \\
\hline & & 6 & 0.160 & 0.229 & $0.010 ; 2.661$ & 0.201 \\
\hline & & 7 & 0.134 & 0.192 & $0.008 ; 2.221$ & 0.161 \\
\hline \multirow[t]{16}{*}{ Geral } & 0 & 0 & 0.157 & 0.116 & $0.037 ; 0.666$ & 0.012 \\
\hline & & 1 & 0.140 & 0.102 & $0.033 ; 0.583$ & 0.007 \\
\hline & & 2 & 0.124 & 0.089 & $0.030 ; 0.510$ & 0.004 \\
\hline & & 3 & 0.110 & 0.081 & $0.026 ; 0.464$ & 0.003 \\
\hline & & 4 & 0.097 & 0.073 & $0.022 ; 0.422$ & 0.002 \\
\hline & & 5 & 0.086 & 0.064 & $0.020 ; 0.366$ & 0.001 \\
\hline & & 6 & 0.075 & 0.055 & $0.018 ; 0.317$ & $<0.001$ \\
\hline & & 7 & 0.065 & 0.049 & $0.015 ; 0.285$ & $<0.001$ \\
\hline & 1 & 0 & 0.217 & 0.147 & $0.058 ; 0.815$ & 0.024 \\
\hline & & 1 & 0.193 & 0.129 & $0.052 ; 0.712$ & 0.014 \\
\hline & & 2 & 0.172 & 0.113 & $0.047 ; 0.622$ & 0.007 \\
\hline & & 3 & 0.152 & 0.102 & $0.041 ; 0.568$ & 0.005 \\
\hline & & 4 & 0.135 & 0.092 & $0.035 ; 0.517$ & 0.004 \\
\hline & & 5 & 0.119 & 0.081 & $0.031 ; 0.449$ & 0.002 \\
\hline & & 6 & 0.104 & 0.698 & $0.279 ; 0.388$ & 0.001 \\
\hline & & 7 & 0.091 & 0.062 & $0.023 ; 0.350$ & $<0.001$ \\
\hline
\end{tabular}

Nota $. \mathrm{ME}=$ meditação; LE = lista de espera; ${ }^{\mathrm{O} O}$ restante foi preenchido como não tendo o desfecho; ${ }^{\mathrm{B}} \mathrm{Razão}$ de odds; ${ }^{\mathrm{C} O s}$ resultados para "Quase exaustão" que deveriam imputar o dado faltante no grupo intervenção como não tendo o desfecho (0) não foram mostrados porque houve problemas de convergência no modelo. 
Vieira, Aguiar, \& Nóbrega, 2009; Paulino, Prezotto, Frias, Bataglia, \& Aprile, 2010), a maior parte dos participantes do presente estudo apresentavam, no pré-teste, a presença de estresse na fase de resistência. De acordo com o modelo quadrifásico de Lipp (Lipp, 2005), as fases do estresse tendem a seguir um processo gradativo de agravamento. Além disso, estudantes com estresse na fase de resistência apresentam uma redução no desempenho acadêmico, bem como um aumento da vulnerabilidade a doenças somáticas (Santos \& Alves Júnior, 2007). Portanto, este tipo de intervenção pode minimizar os efeitos já existentes de estresse, bem como prevenir o seu avanço para a fase de exaustão, a qual é marcada pelo esgotamento psicológico e desenvolvimento de patologias somáticas decorrentes do estresse intenso e contínuo (Lipp \& Malagris, 2001).

Algumas intervenções que ajudaram a reduzir o estresse tiveram duração de quatro semanas (Fan et al., 2014; Greeson et al., 2014; Jain et al., 2007; Shearer, Hunt, Chowdhury, \& Nicol, 2015), sugerindo que formatos mais breves do que o clássico modelo de oito semanas dos protocolos de mindfulness (Khoury et al., 2013) também têm o potencial de produzir mudanças positivas e significativas. Este dado está em consonância com o fato de que os resultados do presente estudo permaneceram significativos mesmo controlando para o número de faltas. Além disso, a obtenção de resultados significativos com um menor número de encontros pode ser especialmente relevante no contexto universitário. Segundo um levantamento realizado com estudantes americanos, entre os principais motivos elencados para justificar a sua baixa procura por serviços e atendimentos psicossociais na fase universitária está a percepção da falta de tempo para realizar este tipo de tratamento (Regehr et al., 2013). A falta de tempo também é considerada um dos fatores desencadeantes do estresse por estudantes brasileiros, tanto de graduação, como pós-graduação (Santos et al., Júnior, 2007).

Portanto, intervenções mais breves podem facilitar uma maior adesão dos estudantes, bem como favorecer uma maior taxa de retenção ao longo da intervenção. Não obstante, é importante que seja mais sistematicamente avaliado se estes estudantes conseguem manter a prática após o término da intervenção. Mesmo que uma metanálise tenha mostrado que os resultados obtidos com intervenções de meditação tendem a manter-se após um período médio de seis meses de follow up (Khoury et al., 2013), não é possível saber qual a proporção de pessoas que de fato mantiveram a prática e se estes efeitos são decorrentes da prática continuada. Além disso, o fato de o presente estudo não ter observado uma diferença entre os grupos na fase de exaustão pode sugerir que seja necessária uma intervenção mais longa para que a mesma produza um efeito significativo sobre as pessoas com níveis severos de estresse. Não obstante, salienta-se que ambos os grupos tiveram redução de sintomas na fase de exaustão no pós-teste. Uma hipótese é que a exaustão, conforme medida pelo ISSL, pode refletir sintomas severos, mas agudos, que diminuem quando a situação causadora dos mesmos cessa. Neste sentido, seria possível considerar que no pré-teste, quando os níveis de exaustão eram maiores para ambos os grupos, os estudantes encontravam-se em algum momento mais exigente do semestre. Já a falta de uma interação significativa entre grupo e tempo para a fase de alerta deve-se ao fato de que no pós-teste nenhum grupo apresentou sintoma nesta fase.

Uma limitação do presente estudo é a avaliação do estresse ter sido feita apenas por autorrelato. Não obstante, já foi demonstrado que a percepção que as pessoas possuem do seu nível de estresse e do seu possível impacto sobre a saúde possui uma associação significativa com a maior probabilidade de apresentar pior saúde e problemas de saúde mental (Keller et al., 2012). Além disso, o efeito da meditação sobre a redução do estresse também é corroborado por estudos que avaliaram parâmetros biológicos. Por exemplo, os níveis de cortisol basal de alunos universitários diminuíram de forma linear e significativa após duas e quatro semanas de um treino de meditação chamado Treinamento Integrativo Mente e Corpo (IBMT), comparado ao grupo controle de relaxamento (Fan et al., 2014). Além disso, este estudo demonstrou que imediatamente após a indução de um estresse agudo a prática do IBMT produziu uma redução significativa da liberação de cortisol, o que não foi observado no grupo de alunos que realizou o relaxamento após a indução de estresse. Resultados análogos também foram obtidos através da mensuração de outro marcador biológico, a variabilidade cardíaca (Shearer et al., 2015). Estudantes universitários que passaram por um treino de quatro semanas de meditação mindfulness apresentaram uma resposta mais adaptativa - indexada pela maior variabilidade cardíaca após uma indução de estresse cognitivo, em comparação a um grupo controle ativo (terapia assistida por animais) e a um grupo controle de lista de espera.

O índice de variabilidade cardíaca pode ser interpretado como um mecanismo psicofisiológico da meditação, sugerindo que a prática ajuda a reduzir a reatividade fisiológica ao evento estressor. Em uma perspectiva psicológica, discute-se que a meditação ajuda a diminuir a atenção voltada a estímulos negativos e consequentemente o seu processamento associativo (Dahl et al., 2015). Tal pressuposto é corroborado por dados mostrando que os níveis de ruminação são menores em meditadores comparado a não meditadores (Guendelman, Medeiros, \& Rampes, 2017). Além disso, os estudos apontam que a prática meditativa está associada a uma maior ativação do córtex cingulado anterior, cuja área é responsável pelo monitoramento de conflitos e pela regulação emocional através do controle cognitivo (Tang, Hölzel, \& Posner, 2015). Também existem evidências de que a meditação ajuda a reduzir a reatividade da amígdala (AM) frente a estímulos emocionais negativos, cujo padrão é precedido por uma maior ativação do córtex pré-frontal dorsomedial e dorsolateral (Lutz et al., 2013). De modo similar, outro estudo mostrou que uma redução da percepção de estresse se correlacionou com uma menor densidade de massa cinzenta na AM direita (Hölzel et al., 2009). Portanto, a meditação parece auxiliar no manejo do estresse devido a uma combinação de mecanismos de regulação atencional e emocional que favorecem que o praticante consiga modular sua percepção e sua resposta às experiências emocionais.

Tomados em conjunto, os dados indicam que a intervenção baseada na meditação pode ajudar na redução do estresse tanto para amostras que não apresentam queixas específicas de estresse (Greeson et al., 2014; Oman et al., 2008; Shapiro et al., 2011), como amostras que encontram- 
se estressadas (exceto quando estão na fase de exaustão) ou que passaram por uma situação de estresse (Fan et al., 2014; Jain et al., 2007; Shearer et al., 2015). A meditação também parece ser um recurso eficaz para condições clínicas de estresse, tal como no Transtorno de Estresse Pós-Traumático (TEPT). Um estudo que avaliou o efeito de duas intervenções - meditação baseada em mantra e meditação mindfulness - em 391 veteranos de guerra com TEPT, encontrou que ambas produziram uma melhora significativa (de efeito moderado) na percepção da severidade dos sintomas em relação ao tratamento usual (Heffner, Crean, \& Kemp, 2016). Este estudo também encontrou que apenas as intervenções de meditação geraram um aumento do escore total de mindfulness medido pela Mindful Attention and Awareness Scale (MAAS), bem como do escore da faceta não reagir às próprias experiências da FFMQ.

No presente estudo, a intervenção de meditação também gerou um aumento significativo dos escores de mindfulness em estudantes universitários, em comparação ao grupo controle, especialmente nas facetas descrever, não reagir e no escore total. Estes resultados corroboram parcialmente achados de estudos anteriores que encontraram mudanças para a faceta descrever e não reagir em estudantes universitários após intervenções baseadas na meditação, comparado a um grupo controle (Baer et al., 2012; Bruin, Meppelink, \& Bögels, 2015; Carmody \& Baer, 2008). No entanto, também existem resultados com universitários que mostraram melhora em outras facetas, tal como observar, não julgar e agir com consciência (Bruin et al., 2015; Carmody \& Baer, 2008). Vale destacar que, assim como descrito na Tabela 2 , no presente estudo o grupo experimental aumentou a média nas facetas observar, não julgar e agir com consciência, o que não foi observado no grupo controle. Assim, uma hipótese para o fato de a interação grupo $\mathrm{x}$ tempo não ter sido significativa para estas facetas é a necessidade de uma intervenção mais longa para obter um efeito significativo nestas habilidades, ou um tamanho amostral maior.

Já com respeito ao escore total de mindfulness, uma metanálise de 93 estudos com adultos (entre estes, universitários) encontrou que as intervenções baseadas na meditação mindfulness produziram uma melhora significativa deste desfecho (Khoury et al., 2013). Esta metanálise (Khoury et al., 2013) também mostrou que o tamanho de efeito da mudança do desfecho de mindfulness moderou o tamanho de efeito das intervenções sobre os desfechos clínicos (sintomas de ansiedade, depressão e estresse). O papel mediador de mindfulness disposicional sobre o desfecho do estresse também foi demonstrado por outro estudo que avaliou amostra universitária (Baer et al., 2012). Estes resultados apoiam a correlação encontrada no presente estudo entre uma menor presença de estresse e uma maior habilidade de mindfulness.

De forma geral, mindfulness compreende uma habilidade de mudarmos o modo como nos relacionamos com nossos pensamentos e sentimentos, especialmente aprendendo a sermos menos reativos aos próprios conteúdos (Baer et al., 2008). Esta premissa está em consonância com o achado de que a percepção que temos do estresse pode ser tão influente na probabilidade de desenvolvermos problemas de saúde física e mental, quanto a presença propriamente dita do estresse (Keller et al., 2012). Assim, do ponto de vista psicológico, o treino da meditação, através do aumento da habilidade de mindfulness, parece ser uma ferramenta que ajuda a mudarmos esta percepção ou a forma como nos relacionamos com ela.

No presente estudo, apenas no grupo meditação uma maior capacidade de descrever relacionou-se com uma maior capacidade de não reagir. Assim, uma hipótese é que nesta amostra, uma das contribuições da meditação foi desenvolver a habilidade de melhor perceber e descrever a si próprio, favorecendo um maior controle sobre a forma de reagir a estes conteúdos. Talvez, o treino de não engajar-se automaticamente no fluxo associativo de ideias - característico da meditação focada -, facilite um maior discernimento de como a atividade mental é gerada e uma maior capacidade de permitir que pensamentos e sentimentos apareçam e desapareçam da consciência, sem que estes produzam, compulsoriamente, alguma reação.

É importante ressaltar que na meditação focada o praticante não deve refletir sobre sua atividade mental ou sobre seus padrões, tampouco interpretá-los, ou intencionalmente modificá-los. O treino envolve apenas focar a atenção na âncora vinculada à respiração, voltando a atenção a este foco sempre que identificar que se distraiu, sem questionar ou elaborar este acontecimento. Assim, naturalmente o praticante desenvolve a habilidade de não envolver-se ou não reagir à própria atividade mental. Portanto, parece que saber reconhecer o padrão mental (por exemplo, automático e ruminativo), modificando a forma como reagimos a ele (habilidades de mindfulness), é um mecanismo importante da meditação. O presente estudo, que avaliou uma meditação focada, ajuda a corroborar o achado de que este mecanismo parece não diferir entre diferentes tipos de meditação sentada e silenciosa (Soler et al., 2014).

Contudo, uma limitação do presente estudo é não ter utilizado um grupo controle ativo, o que impede avaliar se os efeitos sobre os níveis de mindfulness ocorrem pela meditação ou devido a qualquer intervenção psicossocial. Por exemplo, algumas evidências apontam que quando uma intervenção de meditação é comparada a grupos que recebem outro tipo de intervenção (grupo controle ativo), o tamanho de efeito da mudança de mindfulness é menor (Khoury et al., 2013), ou não são observadas diferenças significativas deste desfecho quando as intervenções são comparadas (Goldberg et al., 2015; Goyal et al., 2014). Por outro lado, existem resultados mostrando que meditadores experientes apresentam escores significativamente maiores de mindfulness, em comparação a não meditadores (Baer et al., 2008; Barros et al, 2014; Soler et al., 2014), sendo que esta diferença é mediada pelo tempo e frequência de prática dos meditadores (Soler et al., 2014). Portanto, parece não haver uma uniformidade com relação ao efeito da meditação nas medidas de autorrelato de mindfulness, sendo que alguns autores questionam o grau em que estas medidas, tal como a FFMQ, de fato capturam o construto e refletem aspectos da prática, e discutem que estes instrumentos ainda precisam de mais estudos de validação (Goldberg et al., 2015).

Outras limitações também devem ser consideradas. O número amostral pequeno pode ter reduzido o poder para detectarmos outras mudanças significativas. Não obstante, 
a análise de sensibilidade aumenta a confiabilidade de que o resultado encontrado para o estresse (desfecho primário) é robusto, uma vez que este não teria sido impactado pelos dados faltantes ou por outros possíveis cenários. Em particular, mesmo que o participante da intervenção que não respondeu à avaliação pós-teste estivesse com estresse em alguma das fases e os participantes faltantes do grupo controle não estivessem (pior cenário), o efeito protetivo da intervenção ainda seria encontrado. A não randomização dos participantes é outra potencial limitação, mas as análises da linha de base indicaram que os grupos não diferiram significativamente em nenhuma variável descritiva ou de desfecho. Ainda, o estudo não incluiu um registro das práticas realizadas fora do encontro presencial, impedindo o controle da frequência semanal de prática. Portanto, mesmo que o número de faltas tenha sido controlado nas análises de efeito principal e de interação (GEE), não foi possível controlar um possível efeito moderador da frequência semanal.

Em suma, a despeito de algumas limitações, o presente estudo demonstrou que um treino de seis encontros de meditação focada produziu uma melhora do funcionamento psicológico de estudantes universitários, mesmo com uma média de faltas de 1.55 encontros, relativo ao grupo controle de lista de espera. Em particular, houve uma redução da presença de estresse na fase de resistência, quase exaustão e estresse geral, bem como um aumento das habilidades de mindfulness nas facetas descrever e não reagir, e no escore total. Além disso, os dados corroboram que o aumento de mindfulness pode estar relacionado à redução do estresse. $\mathrm{O}$ grau em que estas mudanças serão mantidas é uma questão de pesquisa que será avaliada em um estudo posterior. O estudo também contribui para o campo na medida em que demonstra que uma intervenção exclusivamente baseada na meditação, e não em um conjunto de distintas práticas - como nos protocolos baseados em mindfulness - também pode produzir benefícios psicológicos para estudantes universitários.

\section{Referências}

Aguiar, S. M., Vieira, A. P. G. F., Vieira, K. M. F., Aguiar, S. M., \& Nóbrega, J. O. (2009). Prevalência de sintomas de estresse nos estudantes de medicina. Jornal Brasileiro de Psiquiatria 58(1), 34-38.

Associação Nacional de Empresas de Pesquisa. (2010). Critério de classificação econômica do Brasil. São Paulo. Recuperado de http://www.anep.org.br/codigosguias/CCEB.pdf

Baer, R. A., Carmody, J., \& Hunsinger, M. (2012). Weekly change in mindfulness and perceived stress in a mindfulness-based stress reduction program. Journal of Clinical Psychology, 68(7), 755-765. https://doi.org/10.1002/jclp.21865

Baer, R. A., Smith, G. T., Lykins, E., Button, D., Krietemeyer, J., Sauer, S., Walsh, E., ... Williams, J. M. (2008). Construct validity of the Five Facet Mindfulness Questionnaire in Meditating and Nonmeditating Samples. Assessment, 15, 329342. doi: 10.1177/1073191107313003

Barros, V. V. D., Kozasa, E. H., Souza, I. C. W. D., \& Ronzani, T. M. (2014). Validity evidence of the brazilian version of the Five Facet Mindfulness Questionnaire (FFMQ). Psicologia: Teoria e Pesquisa, 30(3), 317-327. http://dx.doi.org/10.1590/ S0102-37722014000300009
Beiter, R., Nash, R., McCrady, M., Rhoades, D., Linscomb, M., Clarahan, M., \& Sammut, S. (2015). The prevalence and correlates of depression, anxiety, and stress in a sample of college students. Journal of Affective Disorders, 173, 90-96. https://doi.org/10.1016/j.jad.2014.10.054

Bruin, E. I., Meppelink, R., \& Bögels, S. M. de. (2015). Mindfulness in higher education: Awareness and attention in university students increase during and after participation in a mindfulness curriculum course. Mindfulness, 6(5), 1137-1142. https://doi. org/10.1007/s12671-014-0364-5

Carmody, J., \& Baer, R. A. (2008). Relationships between mindfulness practice and levels of mindfulness, medical and psychological symptoms and well-being in a mindfulnessbased stress reduction program. Journal of Behavioral Medicine, 31(1), 23-33. https://doi.org/10.1007/s10865-0079130-7

Cerchiari, E. A. N. (2004). Saúde mental e qualidade de vida em estudantes universitários (Tese de doutorado não publicada). Universidade Estadual de Campinas (UNICAMP), Faculdade de Ciências Médicas, Campinas, SP. Recuperado de http:// repositorio.unicamp.br/jspui/handle/REPOSIP/313371

Dahl, C. J., Lutz, A., \& Davidson, R. J. (2015). Reconstructing and deconstructing the self: Cognitive mechanisms in meditation practice. Trends in Cognitive Sciences, 19(9), 515-523.

Edwards, L. J. (2000). Modern statistical techniques for the analysis of longitudinal data in biomedical research. Pediatric Pulmonology, 30(4), 330-344.

Fan, Y., Tang, Y., \& Posner, M. (2014). Cortisol level modulated by integrative meditation in a dose-dependent fashion. Stress Health, 30(1), 65-70. https://doi.org/10.1002/smi.2497

Goldberg, S. B., Wielgosz, J., Dahl, C., Schuyler, B., MacCoon, D. S., Rosenkranz, M., ... Davidson, R. J. (2015). Does the Five Facet Mindfulness Questionnaire measure what we think it does? Construct validity evidence from an active controlled randomized clinical trial. Psychological Assessment, 28, 10091014. http://dx.doi.org/10.1037/pas0000233

Goyal, M., Singh, S., Sibinga, E., Gould, N., Rowland-Seymour, A., Sharma, R., Berger, Z.,... Haythornthwaite, J. (2014). Meditation programs for psychological stress and well-being: A systematic review and meta-analysis. JAMA Internal Medicine, 174(3), 357-368.

Greeson, J. M., Juberg, M. K., Maytan, M., James, K., \& Rogers, H. (2014). A randomized controlled trial of koru: A mindfulness program for college students and other emerging adults. Journal of American College Health, 62(4), 222-233. https:// doi.org/10.1080/07448481.2014.887571

Guendelman, S., Medeiros, S., \& Rampes, H. (2017). Mindfulness and emotion regulation: Insights from neurobiological, psychological, and clinical studies. Frontiers in Psychology, 8, 1-23. https://doi.org/10.3389/fpsyg.2017.00220

Heffner, K. L., Crean, H. F., \& Kemp, J. E. (2016). Meditation programs for veterans with posttraumatic stress disorder: Aggregate findings from a multi-site evaluation. Psychological Trauma: Theory, Research, Practice, and Policy, 8(3), 365. http://dx.doi.org/10.1037/tra0000106

Hölzel, B. K., Carmody, J., Evans, K. C., Hoge, E. A., Dusek, J. A., Morgan, L., ... Lazar, S. W. (2009). Stress reduction correlates with structural changes in the amygdala. Social Cognitive and Affective Neuroscience, 5(1), 11-17. https://doi.org/10.1093/ scan/nsp034 
Jain, S., Shapiro, S. L., Swanick, S., Roesch, S., Mills, P., Bell, I., \& Schwartz, G. (2007). A randomized controlled trial of mindfulness meditation versus relaxation training: Effects on distress, positive states of mind, rumination, and distraction. Annals of Behavioral Medicine, 33(1), 12-21. https://doi. org/10.1207/s15324796abm33

Keller, A., Litzelman, K., Wisk, L. E., Maddox, T., Cheng, E. R., Creswell, P. D., \& Witt, W. P. (2012). Does the perception that stress affects health matter? The association with health and mortality. Health Psychology, 31(5), 677. http://dx.doi. org/10.1037/a0026743

Khoury, B., Lecomte, T., Fortin, G., Masse, M., Therien, P., Bouchard, V., ... Hofmann, S. G. (2013). Mindfulnessbased therapy: a comprehensive meta-analysis. Clinical Psychology Review, 33(6), 763-771. https://doi.org/10.1016/j. cpr.2013.05.005

Lipp, M. N. (2005). Manual do inventário de sintomas de stress para adultos de Lipp (ISSL). Casa do Psicólogo, São Paulo.

Lipp, M. N., \& Malagris, L. N. (2001). O stress emocional e seu tratamento. Bernard Range. Artes Médicas, São Paulo.

Lutz, J., Herwig, U., Opialla, S., Hittmeyer, A., Jäncke, L., Rufer, M., ... Brühl, A. B. (2013). Mindfulness and emotion regulation An fMRI study. Social Cognitive and Affective Neuroscience, 9(6), 776-785. https://doi.org/10.1093/scan/nst043

Mari, J. J., \& Williams, P. (1986). A validity study of a psychiatric screening questionnaire (SRQ-20) in primary care in the city of São Paulo. The British Journal of Psychiatry, 148, 23-26. https://doi.org/10.1192/bjp.148.1.23

Menezes, C. B., \& Bizarro, L. (2015). Effects of focused meditation on difficulties in emotion regulation and trait anxiety. Psychology \& Neuroscience, 8(3), 350. http://dx.doi. org/10.1037/pne0000015

Menezes, C. B., Couto, M. C., Buratto, L. G., Erthal, F., Pereira, M. G., \& Bizarro, L. (2013). The improvement of emotion and attention regulation after a 6 -week training of focused meditation: A randomized controlled trial. Evidence-Based Complementary and Alternative Medicine, 2013,1-11. http:// dx.doi.org/10.1155/2013/984678

Menezes, C. B., Fiorentin, B., \& Araujo, L. B. (2012). Meditação na universidade: A motivação de alunos da UFRGS para aprender meditação. Psicologia Escolar e Educacional, 16(2), 309-315.

Neves, M. C. C., \& Dalgalarrondo, P. (2007). Transtornos mentais auto-referidos em estudantes universitários. Jornal Brasileiro de Psiquiatria, 56(4), 237-244. Recuperado de http:// repositorio.unicamp.br/jspui/handle/REPOSIP/21496
Oman, D., Shapiro, S. L., Thoresen, C. E., Plante, T. G., \& Flinders, T. (2008). Meditation lowers stress and supports forgiveness among college students: A randomized controlled trial. Jounal of American College Health, 56(5), 569-578. https://doi. org/10.3200/JACH.56.5.569-578

Paulino, C., Prezotto, A., Frias, A., Bataglia, P., \& Aprile, M. (2010). Sintomas de estresse e tontura em estudantes de pós-graduação. Revista Equilibrio Corporal e Saúde., 2(1), 15-26. http://dx.doi. org/10.17921/2176-9524.2010v2n1p\%25p

Regehr, C., Glancy, D., \& Pitts, A. (2013). Interventions to reduce stress in university students: A review and meta-analysis. Journal of Affective Disorders, 148, 1-11. https://doi. org/10.1016/j.jad.2012.11.026

Santos, A. F., \& Alves Júnior, A. (2007). Estresse e estratégias de enfrentamento em mestrandos de ciências da saúde. Psicologia: Reflexão e Crítica, 20(1), 104-113.

Shapiro, S. L., Brown, K. W., Thoresen, C., \& Plante, T. G. (2011). The moderation of mindfulness-based stress reduction effects by trait mindfulness: Results from a randomized controlled trial. Journal Of Clinical Psychology, 67(3), 267-277. https:// doi.org/10.1002/jclp.20761

Shearer, A., Hunt, M., Chowdhury, M., \& Nicol, L. (2016). Effects of a brief mindfulness meditation intervention on student stress and heart rate variability. International Journal of Stress Management, 23(2), 232.

Soler, J., Cebolla, A., Feliu-Soler, A., Demarzo, M. M., Pascual, J. C., Baños, R., \& García-Campayo, J. (2014). Relationship between meditative practice and self-reported mindfulness: The MINDSENS composite index. PloS one, 9(1), e86622. https://doi.org/10.1371/journal.pone.0086622

Tang, Y. Y., Hölzel, B. K., \& Posner, M. I. (2015). The neuroscience of mindfulness meditation. Nature Reviews Neuroscience, 16(4), 213-225. doi:10.1038/nrn3916

Recebido em 15.03.2016

Primeira decisão editorial em 05.06.2017

Versão final em 04.07.2017

Aceito em 18.09.2017 